\title{
TEMPERATURE EFFECTS IN RHODAMINE B DYES AND IMPROVEMENT IN CW DYE LASER PERFORMANCE
}

\author{
M. A. ALI, J. MOGHADDASI and S. A. AHMED \\ Institute of Ultrafast Spectroscopy and Lasers, Department of Electrical \\ Engineering, The City College of the City, University of New York, \\ New York, NY 10031
}

(Received 6 July, 1990; in final form 18 July, 1990)

\begin{abstract}
The effect of cooling the laser medium on the lasing characteristics of an organic cw dye laser, Rhodamine B in ethylene glycol, pumped by an Argon laser was examined. Significant improvements in the performance of this dye laser were achieved as the solvent temperature was reduced below room temperature, namely: a significant reduction in the threshold power requirements and a considerable increase in the attainable output power. The results obtained may be understood in terms of increased quantum yield of the $\mathrm{RhB}$ dye as the temperature is reduced. This effect overrides possible negative effects that are normally expected due to intermolecular relaxation processes and which are normally observed for other dye lasers which already have high quantum yields at room temperature.
\end{abstract}

\section{INTRODUCTION}

The mechanisms of temperature effects on the fluorescence and absorption spectra of organic dye solutions have received considerable attention. ${ }^{1-3}$ However, the nature of temperature effects on the lasing characteristics of organic dye solutions, when used as a lasing media under laser excitation, is still unclear. ${ }^{4-20}$ This paper examines these effects in laser-pumped cw dye lasers, and shows them to be of considerable practical significance in some special cases.

The optical excitation of a molecule and its subsequent emission of spontaneous luminescence or stimulated radiation results in a series of intermolecular processes (orientational, translational, etc.), which occur in both the ground and excited states. ${ }^{6-11}$ The orientational relaxation processes have a substantial effect not only on the spontaneous luminescence spectrum but also on the stimulated emission spectrum of the solution. ${ }^{15-22}$

To examine these processes, it is necessary, because of the finite duration $\tau_{\mathrm{f}}\left(10^{-8}-10^{-9} \mathrm{~s}\right)$, of the excited states of the molecules studied, to consider the relationship between this quantity and the orientation relaxation time, $\tau_{R}$ $\left(10^{-11}-10^{-12} \mathrm{~s}\right)$, of the surrounding molecules, i.e., to take into account the degree of reorientation of the surroundings, caused by the change in the electric properties of the molecule upon excitation. Under conditions where the role of 
stimulated emission processes predominates, the average time $t$ during which the molecules stay in the excited states decrease sharply and can become comparable to the orientation relaxation time $\tau_{\mathrm{R}}$ for the molecules of the medium at room temperature. In fact, when the threshold pumping level is substantially exceeded, the condition $\tau_{\mathrm{f}} \approx \tau_{\mathrm{R}}$ can be realized in ordinary liquid luminescing solutions at room temperature..$^{15}$ The results of ${ }^{7-10}$ show that at temperatures for which $\tau_{\mathrm{f}} \gg \tau_{\mathrm{R}}$ (total relaxation) or for which $\tau_{\mathrm{f}} \approx \tau_{\mathrm{R}}$ (partial relaxation), energy level scheme for electronic states of complex molecules in polar solvents can be approximated by a model involving four levels, as shown in Figure 1. As a result of intermolecular relaxation processes, which reestablish equilibrium between the excited molecules and the solvent, optically excited molecules decay to an equilibrium level, 3 , with a probability of $\mathrm{P}_{23}=1 / \tau_{\mathrm{R}}$. Then, they emit in the transition $3 \rightarrow 4$, entering the ground Franck-Condon level 4.

Obviously the more rapidly the relaxation processes take place, the greater the probabilities $\mathrm{P}_{23}$ and $\mathrm{P}_{41}$, and the less populated are the final Franck-Condon levels for the absorption and emission events 2 and 4 are as compared with the equilibrium levels 1 and 3. This fact creates favorable conditions for the development of an inverse population of level 3 as compared with level 4 . The gap $\Delta \mathrm{E}_{\mathrm{g}}^{\mathrm{Fc}}$ characterizes the difference in the position of the lower electronic laser level, 4, and the ground state electronic level, which is the original level for absorption (excitation). It has been established ${ }^{16-17}$ that the lasing threshold, as expected,${ }^{18-19}$ is on the whole lower, the greater the value of $\Delta \mathrm{E}_{\mathrm{g}}^{\mathrm{Fc}}$ for a given lasing solution.

In the case of a frozen solution, for which $\tau_{\mathrm{R}} \gg \tau_{\mathrm{f}}$, intermolecular relaxation of orientational type is greatly reduced $\left(\Delta \mathrm{E}_{\mathrm{g}}^{\mathrm{Fc}}=\Delta \mathrm{E}_{\mathrm{e}}^{\mathrm{Fc}} \approx 0\right)$, and the four-level scheme is effectively reduced to a two-level scheme. Mathematical expressions for $\Delta \mathrm{E}_{\mathrm{g}}^{\mathrm{Fc}}$ as well as the whole theoretical treatment of the theory of universal intermolecular interactions, given in the literature,$^{11-15}$ are very complicated and in our opinion cannot be taken to be complete. Extensive experimental and theoretical data, ${ }^{16-2}$ devoted to uncover temperature effects on the lasing characteristics of dye solutions, when used as a lasing media under laser excitation, reveal that in the initial stage of cooling there is a slight decrease in the threshold pumping intensity, and then with further cooling, as a rule, threshold requirements begin to increase greatly, leading

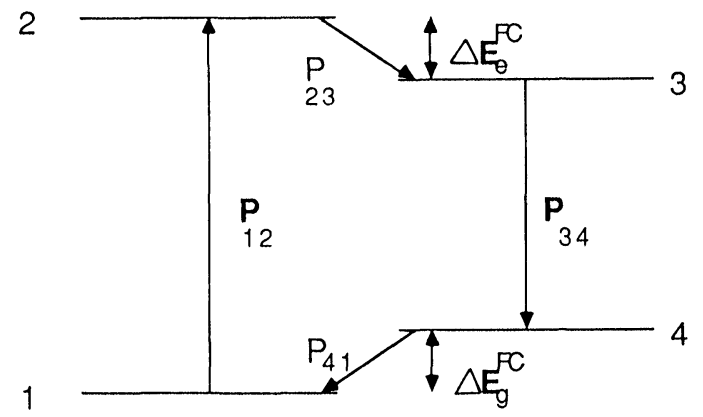

Figure 1 Energy level scheme for electronic states of complex dye molecules in a polar solvent. 
finally to the collapse of lasing $\left(\tau_{\mathrm{R}} \gg \tau_{\mathrm{f}}\right.$ and $\left.\Delta \mathrm{E}_{\mathrm{g}}^{\mathrm{Fc}} \approx 0\right)$. While the theory of universal intermolecular interactions for the two limiting cases, $\tau_{\mathrm{f}} \gg \tau_{\mathrm{R}}$ and $\tau_{\mathrm{R}} \gg \tau_{\mathrm{f}}$, is in good agreement with the experimental results, it cannot explain the reduction in threshold pumping intensity in the initial stage of cooling, neither can it give satisfactory results in the case of a gradual transition from a four-level scheme to two-level scheme in the arrangement of the electronic levels.

The present work is devoted to a further study of temperature effects on the lasing characteristics of dye solutions, when used as a lasing media under laser excitation, particularly at the initial stage of cooling $\left(\mathrm{T} \approx 23^{\circ} \mathrm{C}\right.$ to $\left.-5^{\circ} \mathrm{C}\right)$ where the theory of universal intermolecular interactions cannot be used to interpret the experimental results. We report experimental results of the temperature effect on the cw lasing and fluorescence characteristics of Rhodamine B in ethylene glycol for different concentrations.

\section{EXPERIMENTAL: RESULTS AND ANALYSIS}

The temperature dependence of fluorescence and lasing characteristics of cw circulating dye laser, excited with the multimode output of an Argon laser, were examined for Rhodamine B laser solutions in ethylene glycol. The laser pumped cw dye laser geometry is the customary longitudinal arrangement used in almost all cw dye lasers. ${ }^{3}$ The dye laser cavity employed here is shown in Figure 2. The cavity is a pair of $7.5 \mathrm{~cm}$ radius mirrors in a confocal arrangement, which requires four transits of the cavity for the path of the beam to close, giving a $1 \mathrm{GHz}$ cavity mode spacing. The cavity is used off-axis, that is with the two output spots not overlapping to avoid the variation with microphonic motions that the maximum transmission through the cavity would undergo if the spots were allowed to overlap and interfere. The correct

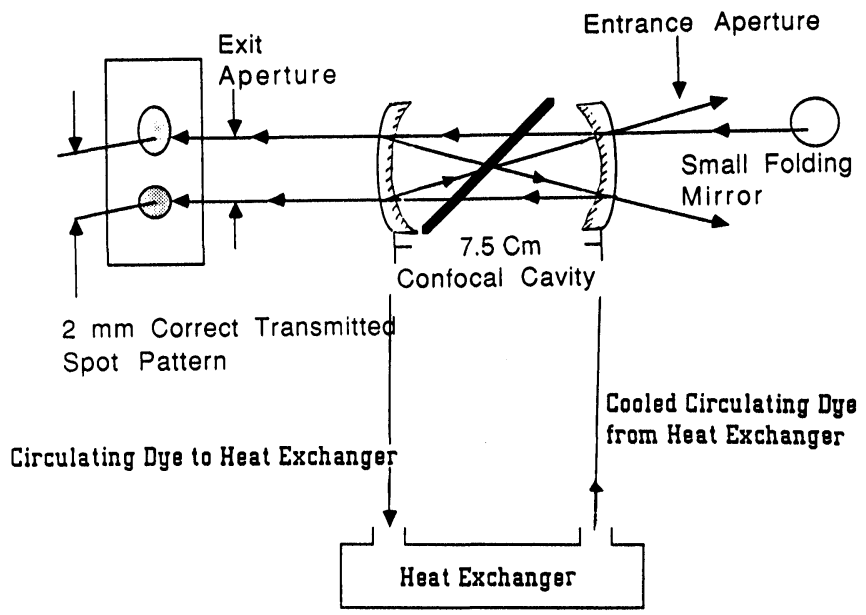

Figure 2 Experimental configuration of the dye laser system. 
separation of the spots is approximately $2 \mathrm{~mm}$ between centers. The oscillator cavity also contains a diffraction grating for tuning. The solution was circulated through a reservoir, the dye cell, and a coiled heat exchanger. Liquid nitrogen was used for cooling of the solution in the circulating reservoir.

Figure 3 shows typical behavior of the fluorescence spectra, at various temperatures, for RhB solution in ethylene glycol. The fluorescence spectra shown in Figure 3 were excited with the multimode output of an Argon laser. As expected, the fluorescence spectra narrow with decreasing temperature, however, there is no appreciable shift in peaks. As shown in Figure 3, as the temperature decreases, there is a sharp increase in the fluorescence quantum yield.

The temperature dependence of the lasing characteristics were obtained for four different concentrations, $2 \times 10^{-4}, 3 \times 10^{-4}, 4 \times 10^{-4}$, and $5 \times 10^{-5} \mathrm{moles} / \mathrm{liter}$ using broadband mirrors for the dye laser.

The experimental results for the temperature dependence of the lasing threshold and lasing output (for constant excitation) are shown in Figures 4 and 5, respectively. It can be shown from Figure 4 that in the initial stage of cooling, for all concentrations, as $T$ drops from $23^{\circ} \mathrm{C}$ to $-5^{\circ} \mathrm{C}$, there is a considerable reduction in the lasing threshold. It can also be seen from Figure 4 that the reduction is more significant at a

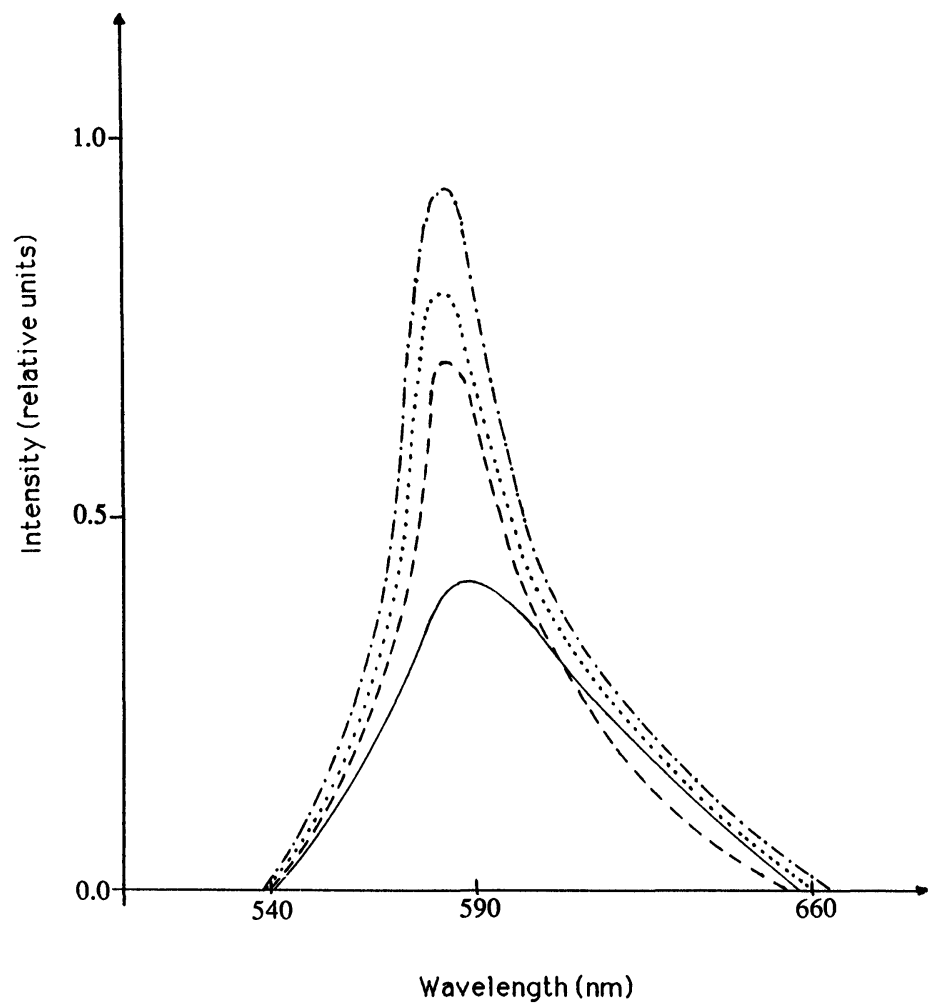

Figure 3 Fluorescence spectra of Rhodamine B in ethylene glycol. $-23^{\circ} \mathrm{C}, \cdots-5^{\circ} \mathrm{C}, \cdots-20^{\circ} \mathrm{C}$ and $\cdots-60^{\circ} \mathrm{C}$. 


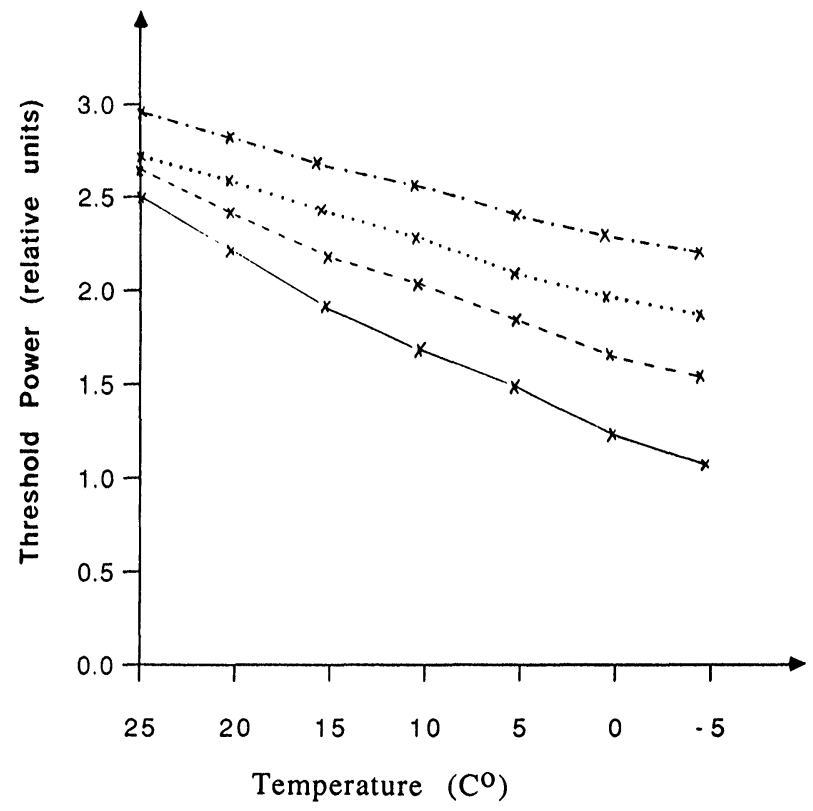

Figure 4 Lasing threshold pumping intensity Vs solution temperature for multimode regime. $3 \times 10^{-4} \mathrm{M} / \mathrm{L} \ldots 2 \times 10^{-4} \mathrm{M} / \mathrm{L} \cdots 4 \times 10^{-4} \mathrm{M} / \mathrm{L}$ and $\cdots . .5 \times 10^{-5} \mathrm{M} / \mathrm{L}$.

concentrations of $3 \times 10^{-4}$ moles/liter. This significant reduction in the lasing threshold cannot be explained in the framework of a model of universal intermolecular interactions which, as a general rule, predict an increase in the lasing threshold with reduction in temperature. ${ }^{15-22}$ However, as shown in Figure 3, this significant reduction in the lasing threshold is explained by the fact that as the temperature is reduced the value of the quantum yield of spontaneous luminescence sharply increases, overriding any possible negative effects due to intermolecular relaxation processes. As might be expected, as shown in Figure 5, at temperature of $-5^{\circ} \mathrm{C}$ and concentrations of $3 \times 10^{-4}$ moles/liter, where the lasing threshold is minimum, the lasing output power also reaches a maximum. Because of circulation requirements, it was not practical to cool the dye solution below $-5^{\circ} \mathrm{C}$ in the $\mathrm{cw}$ laser arrangement. However, it can be expected that with further cooling $\left(T<-5^{\circ} \mathrm{C}\right)$, negative effects due to intermolecular relaxation processes would begin to assert themselves. ${ }^{16-22}$ Thus, despite the continuous increase in the fluorescence quantum yield observed with decreasing temperature (Figure 3), it can be expected that eventually the lasing threshold would again increase, leading finally to the collapse of lasing $\left(\tau_{\mathrm{R}} \gg \tau_{\mathrm{f}}\right.$ and $\left.\Delta \mathrm{E}_{\mathrm{g}}^{\mathrm{Fc}} \approx 0\right) .{ }^{16-19}$

To further examine the temperature dependence of the lasing characteristics, measurements of the lasing threshold and output power were repeated, at two different temperatures, for a range of laser output wavelengths (using a prism for selectivity). These measurements are shown in Figures 6 and 7 for dye solution concentrations of $2 \times 10^{-4}$ and $3 \times 10^{-4}$ moles/liter, respectively. As expected, for 


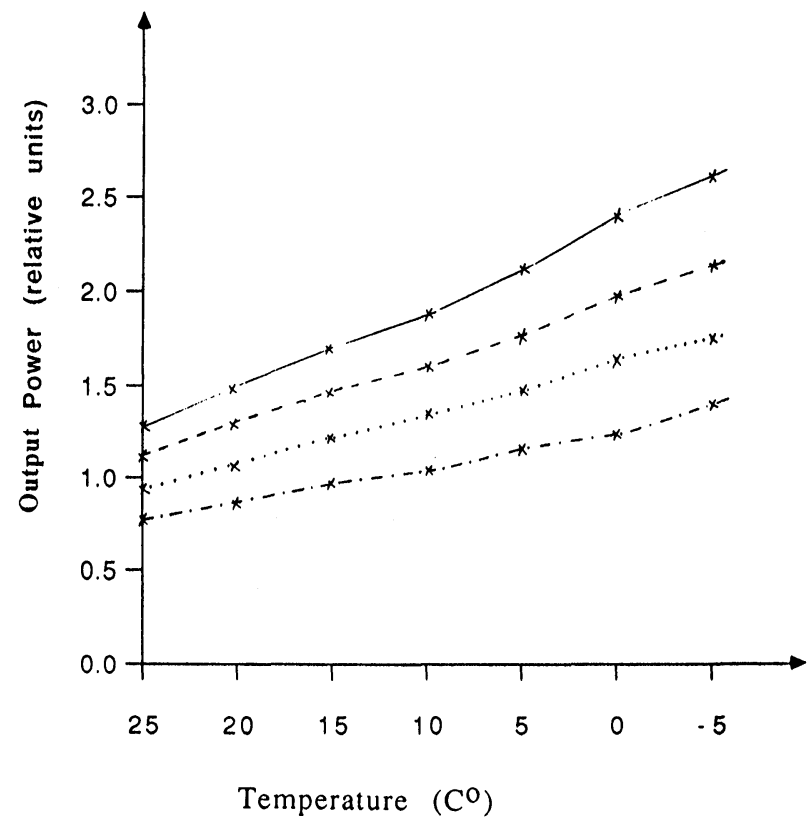

Figure 5 Lasing output power Vs solution temperature for multimode regime. $3 \times 10^{-4} \mathrm{M} / \mathrm{L}$ $\ldots 2 \times 10^{-4} \mathrm{M} / \mathrm{L} \cdots 4 \times 10^{-4} \mathrm{M} / \mathrm{L}$ and $\ldots . .5 \times 10^{-5} \mathrm{M} / \mathrm{L}$.

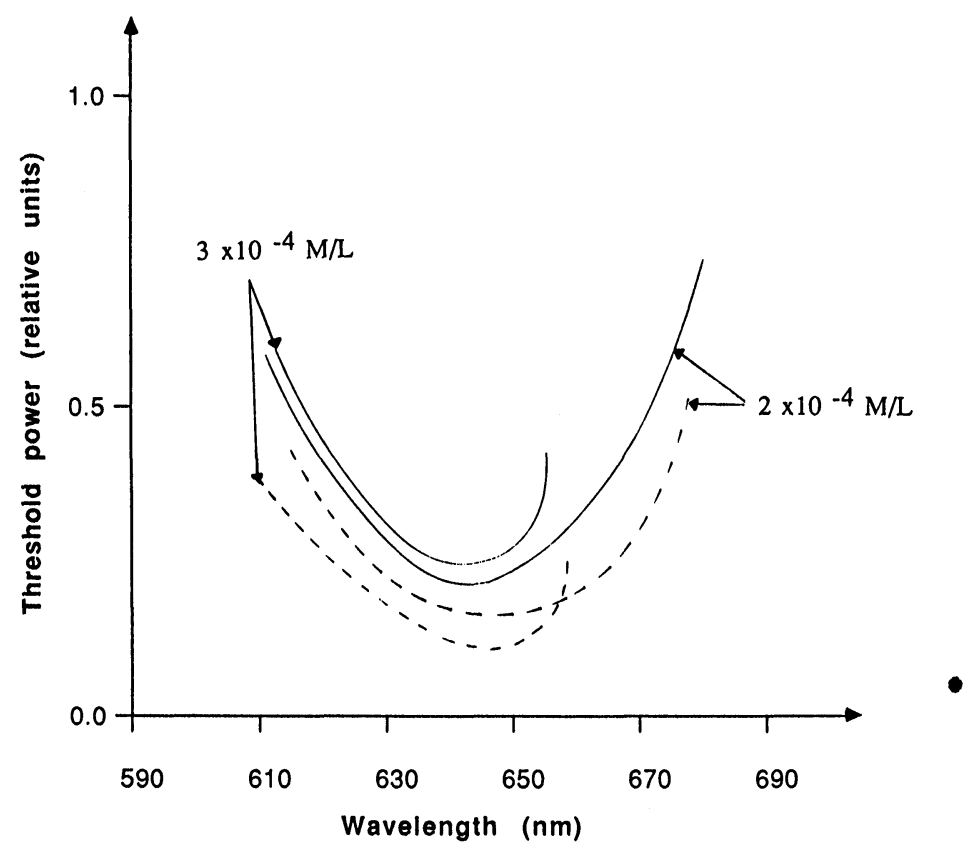

Figure 6 Lasing threshold pumping intensity Vs wavelength for single-frequency regime. $--T=-5^{\circ} \mathrm{C}$ and $-T=23^{\circ} \mathrm{C}$. 


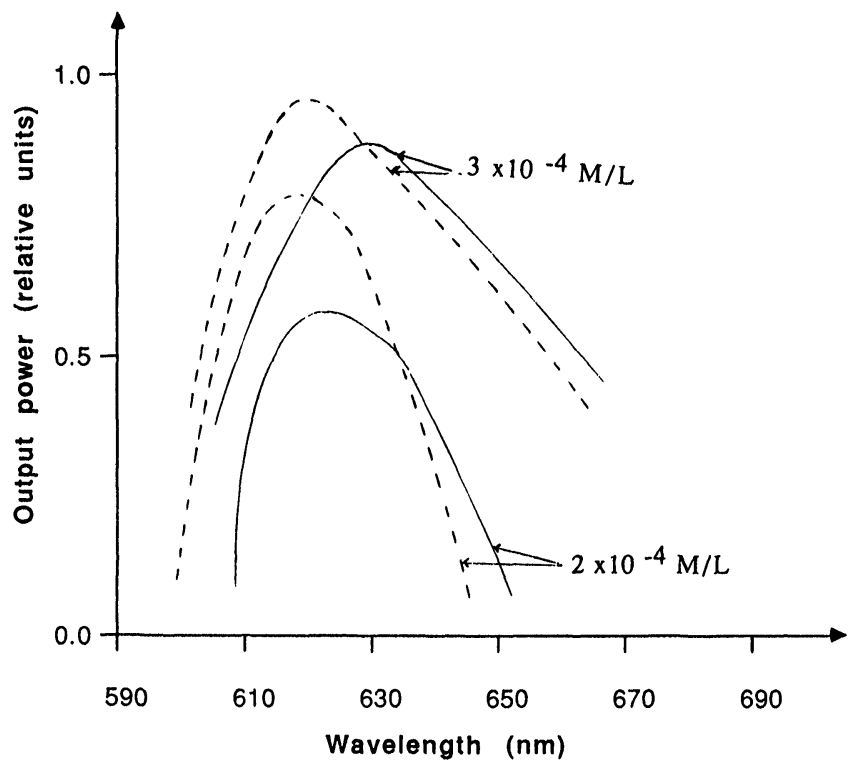

Figure 7 Lasing output power Vs wavelength for single-frequency regime. - $-T=-5^{\circ} \mathrm{C}$ and $T=23^{\circ} \mathrm{C}$.

all wavelengths throughout the entire spectrum of interest, lasing threshold and output power were significantly improved at $-5^{\circ} \mathrm{C}$ compared to values measured at $23^{\circ} \mathrm{C}$.

It should be pointed out that these results are in contrast to the results obtained for most other dye solutions (e.g. solutions of Rhodamine $6 \mathrm{G}$ in methanol, Rhodamine $\mathrm{C}$ in methanol and 3-amino-N-methylphthalimide in glycerol), where quantum yields are already high at room temperature, and where cooling has initially little or no impact, and further cooling results in reduction in laser output and increase in threshold requirements, in conformity with the theory of universal intermolecular interactions. $^{20-23}$

Finally, it should be added that, although our work have mainly focused on the two most important laser parameters, namely: output power and threshold power requirements. However, other important laser parameters, such as beam divergence and frequency stability, depend largely on the thermal stability of the laser cavity. In fact, during the course of cooling the solution, the observed increase in the laser output power was accompanied by as much as a $12 \%$ decrease in the divergence angle of the laser beam. The divergence angle was determined from the far-field image at a distance of $15 \mathrm{~m}$ from the dye cell with a precision of $\pm 0.5 \mathrm{mrad}$. The decrease in the divergence angle of the laser beam may be explained by a decrease in thermally induced refractive index gradients. ${ }^{24}$ With respect to wavelength stability, for a $10^{\circ} \mathrm{C}$ increment in temperature, a $0.1 \mathrm{~cm}^{-1}$ wavelength shift should be expected. ${ }^{25-26}$ In our experiments, a slightly higher effect, $0.13 \mathrm{~cm}^{-1}$ wavelength shift, was noticed. The frequency shift is almost entirely attributed to variations in 
the physical disposition of the cavity components, especially the grating and the output coupler mirror. ${ }^{26}$

In conclusion, it has been shown that, for the case of RhB-ethylene glycol laser solution, cooling from room temperature to $-5^{\circ} \mathrm{C}$ can result in significant increases in cw dye laser output, and related reductions in threshold power requirements. These results are explained by the fact that as the temperature is reduced the value of the quantum yield of spontaneous luminescence for $\mathrm{RhB}$ sharply increases, overriding possible negative effects due to intermolecular relaxation processes. These results demonstrate that an understanding of the temperature effect on the spectrum and lasing efficiency of dye solutions requires an explanation beyond the framework of the theory of universal intermolecular interactions, particularly at initial stages of cooling below room temperature.

\section{References}

1. Joseph R. Lakowicz, Principles of fluorescence spectroscopy, third edition (Plenum Press, New York, 1968).

2. M. U. Belyi and A. B. Leontev, Opt. Spektrosc. 34, 715-721 (1972).

3. F. P. Schafer, Dye Lasers, Second Revised Edition, (Springer-Verlag, Berlin, Heidelberg, New York 1977).

4. N. G. Bakhshiev and Yu. T. Mazurenko, Opt. Spectrosc. 25 (1970).

5. N. G. Bakshiev and I. V. Piterskaya, Opt. Spectrosc. 16 (1966).

6. V. P. Klochkov and S. M. Korotkov, Opt. Spectrosc. 25 (1970).

7. A. V. Aristov and Yu. S. Maslyukov, Opt. Specrosc. 18 (1968).

8. N. G. Bakshiev, Opt. Spectrosk. 32, 1151 (1972).

9. N. G. Bakshiev, O. P. Grin and V. I. Studenov, Opt. Spectrosk. 39, 54-59 (1975).

10. N. G. Bakshiev, Opt. Spectrosk. 32, 979 (1972).

11. V. A. Alekseev, L. K. Denisov, V. I. Kozintsev and N. A. Kozlov, J. Appl. Spectrosc. 31, 848 (1979).

12. L. V. Levshin, T. D. Slavnova, and V. I. Yuzhakov, J. Appl. Spectrosc. 24, 698 (1976).

13. V. I. Studenov, N. G. Bakhshiev, and V. S. Smirnov, Reports of the 7th-All-Union Conference, Tashkent (1974).

14. A. N. Rubinov and V. I. Tomin, Opt. Spektrosk. 29, 1802 (1970).

15. A. V. Aristov, N. G. Bakhshiev and I. V. Piterskaya, Opt. Spektrosk. 30, 143 (1971).

16. V. I. Studenov and N. G. Bakhshiev, Opt. Spektrosk. 36, 392 (1974).

17. V. I. Studenov, I. V. Piterskaya and N. G. Bakhshiev, Opt. Spektrosk. 39, 308 (1975).

18. N. G. Bakhshiev, The Spectroscopy of Intermolecular Interactions (Nauka, Leningrad, 1972).

19. N. G. Bakshiev, Opt. Spectrosk. 32, 1151 (1972).

20. N. G. Bakhshiev and V. I. Studenov, Opt. Spektrosc. 33, 62-65 (1972).

21. V. I. Studenov and N. G. Bakhshiev, Opt. Spektrosk. 39, 661-665 (1975).

22. N. V. Korol' Kova and B. M. Uzhinov, Zhurnal Prikladnoi Spektroskopii, 39(3), 406-412 (1983).

23. V. M. Krasnoshchekov, A. B. Nikolaev and V. V. Rylkov, Opt. Spektrosk. 54, 118-122 (1983).

24. D. W. Peters and C. W. Mathews, Appl. Opt. 19, 4131 (1980).

25. D. C. Hanna, P. A. Karkkainen and R. Wyatt, Opt. Quantum Electron. 7, 115-119 (1975).

26. F. J. Duarte, IEEE J. Quantum Electron. QE-19, 1345-1347 (1983). 